\title{
On the choice of coupling procedure for the Poincaré gauge theory of gravity
}

\author{
Marcin Kaźmierczak \\ Institute of Theoretical Physics, Uniwersytet Warszawski, Hoża 69, 00-681 Warszawa, Poland
}

\begin{abstract}
The gauge approach to the theory of gravity has been widely discussed as an alternative to standard general relativity. The Poincaré group, as a symmetry group of all relativistic theories in the absence of gravitation, constitutes the most natural candidate for a gauge group. Although the Poincaré gauge theory of gravity has been elaborated over the years and cast into a beautiful formal framework, some fundamental problems have remained unsolved. One of them concerns the inclusion of matter. The minimal coupling procedure, which is employed in standard Yang-Mills theories, appears to be ambiguous in the case of gravity. We propose a slight modification of this procedure, which removes the ambiguity. Our modification justifies some earlier results concerning the consequences of the Poincaré gauge theory of gravity. In particular, the predictions of EinsteinCartan theory with fermionic matter are rendered unique. We recall the earlier proposed solution based on modified volume-forms. The advantage of our modification is that the predictions of the theory are not radically changed. Basically, this modification simply justifies the results that were obtained partly 'by chance' in the hitherto prevailing accounts on the Einstein-Cartan theory. The only difference in the predictions, when compared to the standard treatment, concerns the Proca field in the presence of gravity. The 'torsion singularities' which occur there are shifted towards other values of the field.
\end{abstract}

PACS numbers: 04.50.Kd, 04.40.-b, 11.30.Er, 11.15.-q

\section{INTRODUCTION}

As noted by Einstein himself [1, the theory of gravity can be formulated by considering the metric and the connection as independent fields, which is referred to as first order formalism. It was originally observed by Weil [2] that this formulation leads to different predictions, when compared to the standard second order metric approach, after fermions are included. At the same time, Weil pointed out that the second order formalism would yield the results indistinguishable from those produced by the first order one if fermionic Lagrangian was supplemented by an additional term. However, this term would have to contain fourth order powers of the Dirac field and the flat space fermionic Lagrangian thus obtained would generate a nonlinear equation, instead of the standard Dirac one. It is also important to stress that Weil concentrated on the equations for the metric and the Dirac field. He would not be able to state an equivalence of the two formalisms if he attached any significance to the space-time torsion.

Since the introduction by Yang and Mills of the non-Abelian gauge theories [3], attempts have been undertaken of describing all the known interactions as emerging from the localization of some fundamental symmetries of the laws of physics. It is now clear that all the non-gravitational fundamental interactions can be successfully given such an interpretation. The Yang-Mills theories constitute a formal basis for the standard model of particle physics. However, gravitational interaction has always been an odd one. Although the attempts to describe gravity as a gauge theory were initiated by Utiyama [4 within a mere two years after the pioneering work of Yang and Mills, the construction of this theory seems yet not to be satisfactorily completed. All the relativistic theories in the absence of gravity are invariant under the (global) action of the Lorenz group. They are also trivially invariant under space-time translations. Therefore, it seems natural to adopt either the Lorenz group or the full Poincaré group as a gauge group. Utiyama's approach employed the Lorenz group, but then it was necessary to introduce ad hoc a set of fields, which were subsequently identified with the tetrad system on the resulting Riemannian manifold. It was then observed by Kibble [5] that this and other failings of Utiyama's theory can be remedied if the full Poincaré group is promoted to the gauge group. This necessitates the first order formalism for general relativity, with metric (but non-symmetric) connection, as the set of Yang-Mills fields has to consist of ten independent one-forms. At the same time, another aesthetic deficiency of GR was removed. At the microscopic level, elementary particle states can be classified by the irreducible unitary representations of the (universal covering of the) Poincaré group, which are labeled by mass and spin of the particle. It seems rather artificial to assume that distribution of masses produces the curvature of space-time, whereas spin does not influence the geometry at all. In the Poincaré gauge theory, the macroscopically

*Electronic address: marcin.kazmierczak@fuw.edu.pl 
averaged distribution of spin appears to be a source of the space-time torsion. This fact was in agreement with the earlier ideas of Elié Cartan and hence the resulting modification of GR is usually referred to as Einstein-Cartan theory. The curvature and the torsion of space-time are parts of the curvature of the Poincaré connection of the underlying gauge theory, which is explained in Section II.

If a field theory in Minkowski space is given, this theory being symmetric under the global action of a representation of a Lie group, the natural way to introduce the corresponding interaction within the spirit of Yang-Mills theory is to apply the minimal coupling procedure (MCP). Indeed, in the standard model of particle physics this procedure is followed on the fundamental level, leading to predictions that agree with experimental results with great accuracy. In GR, the principle of equivalence, which states that the effects of gravitation can be locally 'turned off' by a suitable choice of a reference frame, necessitates minimal coupling. This principle alone can be used to derive a majority of predictions of GR (see 6] and Appendix VIII), which are again excellently confirmed by observations and experiments. However, trying to apply MCP in order to pass from a field theory in flat space to a Riemann-Cartan space-time ${ }^{1}$ results in difficulties. This is because adding a divergence to the flat space Lagrangian density, which is a symmetry transformation, leads to the non-equivalent theory in curved space after MCP is applied. Although this problem was observed already by Kibble, it has been largely ignored in the subsequent investigations concerning EC theory. The paper [7] provides an interesting example. The authors begin with a particular flat space fermionic Lagrangian, which is quadratic in fields and generates the Dirac equation. Then MCP is applied and standard gravitational (first order) term is added to the Lagrangian. Owing to the algebraic character of the equation that relates the density of spin and the torsion, the latter can be expressed through matter fields and the result can be inserted back into the Lagrangian. In this way an effective action is obtained that depends on the metric and the matter fields only. Its gravitational part is represented by the standard second order action of GR. The matter part, however, differs from the one that would be obtained via MCP in standard GR from the same initial flat space fermionic Lagrangian. The difference is represented by an additional term, which is the square of the Dirac axial current, preceded by a very small coupling constant (of order $l^{2}$, where $l$ is the Planck length). This term can be interpreted as describing a gravitationallyinduced point-interaction between fermions. Further, one can find the equation for the Dirac field, which is nonlinear, even in the limit of the space-time metric being Minkowski's flat one. Although derivations presented in [7] were slightly different from the line of reasoning presented above, the existence of such a weak contact interaction in EC theory was the main result of the paper in question. But is it justified to claim that the occurrence of this interaction distinguishes the EC theory from GR? In fact, one could find the Minkowskian limit of the matter part of the effective Lagrangian and use the resulting modified fermionic Lagrangian as a starting point for MCP in GR. One would than trivially obtain the same curved space action as the effective one of EC theory. Hence, as far as torsion is not given a fundamental significance, the two theories (EC and GR) could be considered as indistinguishable. This is exactly what Weil concluded already in 2. However, in GR we need to put the point interaction into the theory by hand, whereas in EC it arises naturally as a consequence of an interrelation between spin and torsion. If we postulated that a simple (second order in field powers) Lagrangian generating standard Dirac equation should be used as a starting point for MCP in each case (one could discuss whether such a postulate is justified or rather artificial), then it could seem to follow from the analyses of [2] and [7] that EC gravity indeed differs from GR by the presence of the above discussed point fermion interaction.

Unfortunately, even such a statement, after all the stipulations that we have made, would not be true. It is namely important which simple Lagrangian generating Dirac equation is used. In flat space, we are free to add a divergence of a vector field to the Lagrangian density, which would make difference for the final theory in the Riemann-Cartan space, as mentioned above. Exploiting this freedom, one can produce, in addition to the axial-axial point-interaction, also a vector-vector one, as well as a parity breaking axial-vector one. The coupling constants of these interactions may reach significant values. What is more, one can eliminate all of them on the effective level and thus render the predictions of EC theory with fermions indistinguishable from the predictions of GR, in spite of all our limitations. These facts were discussed in [8]. Hence, it seems that MCP should be somehow modified for the sake of connections with torsion, so that it gives equivalent results for equivalent flat space Lagrangians. This issue is of principal importance if EC theory is to regain its predictive power. An attempt to establish such a modified procedure was made by Saa [9] [10. Unfortunately, Saa's solution results in significant departures from standard GR, which seem incompatible with observable data [11] 12, unless some additional assumptions of rather artificial nature are made, such as demanding a priori part of the torsion tensor to vanish [13. The main purpose of this paper is to introduce an alternative modification of MCP, which also eliminates the ambiguity. Unlike Saa's proposal, our approach does not lead to radical changes in the predictions of the theory. In the case of gravity with fermions, the procedure simply justifies the earlier results of [7, 14, 15, 16, 17. These results were obtained partly 'by chance', as the flat space Dirac

\footnotetext{
${ }^{1}$ A Riemann-Cartan space is a manifold with a metric tensor and a metric connection (in general non-symmetric).
} 
Lagrangian was randomly selected from the infinity of equally good possibilities. The same applies to the paper by Perez and Rovelli [18 in which the semi-classical physical effects of the Immirzi parameter of Loop Quantum Gravity were discussed. As shown in [19, the predictions of EC gravity with Holst term and fermions radically change when we pass from one equivalent fermionic Lagrangian to another. However, if we use the corrected coupling procedure proposed here, instead of the standard MCP, the two-parameter family of theories of [19] shrinks into the unique theory, which is exactly the one described in [18.

After the Poincare gauge theory was brought to life by Kibble, the relation between the translational gauge fields and the tetrad from the point of view of fibre bundle formulation was clarified by Trautman [20] (see also [21]). There the whole affine group was considered as a gauge group. The geometric concept of a radius mapping, which was used in 20, is intimately connected with the Poincaré coordinates, whose physical meaning was later elaborated in 22. For an exhaustive review of possible approaches to the formulation of gauge theory of gravity, see [23. In this paper we aim to formulate the theory in a possibly simple (though precise) manner, rather than to acquire the highest degree of generality and mathematical complexity. Such a simple formulation is presented in Section III. In Section III we explain the origin and consequences of the ambiguity of MCP in the presence of torsion, using the Dirac field case as an instructive example. In Section IV we present two possible modifications of MCP which remove the ambiguity. First of them is the earlier Saa's procedure and the second is our proposal. We comment on the aesthetical advantages of a Poincaré gauge formulation of gravity combined with our coupling procedure. In Section $\mathrm{V}$ we describe the consequences of application of our procedure to different field theories. An interesting issue is the fate of torsion singularities in the theory of Proca field. Finally in Section VI we draw the conclusions.

\section{THE POINCARÉ GAUGE THEORY}

\section{A. Yang-Mills theories}

Let us recall the classical formalism of a Yang-Mills gauge theory of a Lie group $G$. Let

$$
S[\phi]=\int \mathcal{L}\left(\phi, \partial_{\mu} \phi\right) d^{4} x=\int \mathfrak{L}(\phi, d \phi)
$$

represent the action of a field theory in Minkowski space $M$. Here $\mathcal{L}$ is a Lagrangian density and $\mathfrak{L}$ a Lagrangian four-form. Assume that $\mathcal{V}$ is a (finite dimensional) linear space in which fields $\phi$ take their values, $\phi: M \rightarrow \mathcal{V}$, and $\pi$ is a representation of $\operatorname{Lie}(G)$ on $\mathcal{V}$. Let $\rho$ denote the corresponding representation of the group ${ }^{2}, \rho(\exp (\mathfrak{g}))=\exp (\pi(\mathfrak{g}))$. Suppose that the Lagrangian four-form, and hence the action, is invariant under its global action

$$
\mathfrak{L}(\rho(g) \phi, d(\rho(g) \phi))=\mathfrak{L}(\rho(g) \phi, \rho(g) d \phi)=\mathfrak{L}(\phi, d \phi) .
$$

Then one can introduce an interaction associated to the symmetry group $G$ by allowing the group element $g$ to depend on space-time point and demanding the theory to be invariant under the local action of $G$. This can be most easily achieved by replacing the differential by the covariant differential

$$
d \phi \rightarrow D \phi=d \phi+\mathbb{A} \phi,
$$

where $\mathbb{A}$ is a $\operatorname{Lie}(G)$-valued ${ }^{3}$ one-form field on $M$ which transforms under the local action of $G$ as

$$
\mathbb{A} \rightarrow \mathbb{A}^{\prime}=\rho(g) \mathbb{A} \rho^{-1}(g)-d \rho(g) \rho^{-1}(g) .
$$

Then $D^{\prime} \phi^{\prime}=\rho(g) D \phi$ and $\mathfrak{L}(\phi, D \phi)$ is invariant under the local action of $G$, on account of (II.2). If the basis of $\operatorname{Lie}(G)$ is chosen, the components of $\mathbb{A}$ represent Yang-Mills fields of the resulting theory. The field strength can be represented by a $\operatorname{Lie}(G)$-valued two-form

$$
\mathbb{F}:=d \mathbb{A}+\mathbb{A} \mathbb{A}
$$

which transforms in the adjoint representation of $\operatorname{Lie}(G)$ and obeys Bianchi identity

$$
\mathbb{F}^{\prime}=\rho(g) \mathbb{F} \rho^{-1}(g), \quad d \mathbb{F}+[\mathbb{A}, \mathbb{F}]=0 .
$$

Think of $\mathbb{A}$ and $\mathbb{F}$ as matrices belonging to the representation of $\operatorname{Lie}(G)$ whose entries are one-forms on $M$. When multiplying such matrices, one should multiply their entries externally. The next step is the construction of a gaugefield part of the action, which should be built of $\mathbb{A}$ and remain invariant under gauge transformations (II.4).

\footnotetext{
2 More precisely, in a generic case $\rho$ is a representation of the universal covering group of $G$, which may not be a representation of $G$ itself.

${ }^{3}$ More precisely, $\mathbb{A}$ and $\mathbb{F}$ take values in the representation $\pi$ of $\operatorname{Lie}(G)$. The same concerns similar situations appearing later on.
} 


\section{B. The Poincaré group as a gauge group}

The Poincaré group $\mathcal{P}$ consists of all isometries of Minkowski space. They can be represented by pairs $(\Lambda, a)$ acting on $M$ as $(\Lambda, a) x=\Lambda x+a$, where $x \in M, a$ represents a column of four real numbers and $\Lambda \in O(1,3)$ is a Lorenz matrix ${ }^{4}$. One easily finds out that the composition law is $\left(\Lambda_{1}, a_{1}\right)\left(\Lambda_{2}, a_{2}\right)=\left(\Lambda_{1} \Lambda_{2}, \Lambda_{1} a_{2}+a_{1}\right)$. Let

$$
\begin{aligned}
& \rho(\Lambda, a):=\rho(a) \rho(\Lambda), \\
& \rho(a):=\exp \left(a_{a} P^{a}\right), \quad \rho(\Lambda(\varepsilon)):=\exp \left(\frac{1}{2} \varepsilon_{a b} J^{a b}\right)
\end{aligned}
$$

be the representation of $\mathcal{P}$. Here $P^{a}, J^{a b}$ are the generators of translations and Lorenz rotations and belong to the representation $\pi$ of $\operatorname{Lie}(\mathcal{P})$ (see previous subsection). The coefficients $\varepsilon_{a b}=-\varepsilon_{b a}$ are parameters of the Lorenz transformation $\Lambda(\varepsilon)=\exp (\varepsilon)$. Here $\varepsilon \in s o(1,3)$ is the matrix with entries $\varepsilon_{b}^{a}:=\eta^{a c} \varepsilon_{c b}$, where $\left(\eta^{a b}\right)=\left(\eta_{a b}\right)=$ $\operatorname{diag}(1,-1,-1,-1)$ is Minkowski matrix. Using the composition law and employing infinitesimal transformations one can derive transformation properties of the generators and the commutation relations for the Poincaré algebra (see [30] for these derivations)

$$
\begin{aligned}
& \rho(\Lambda, a) P^{a} \rho^{-1}(\Lambda, a)=\Lambda_{c}{ }^{a} P^{c}, \\
& \rho(\Lambda, a) J^{a b} \rho^{-1}(\Lambda, a)=\Lambda_{c}{ }^{a} \Lambda_{d}{ }^{b}\left(J^{c d}+a^{c} P^{d}-a^{d} P^{c}\right), \\
& {\left[P^{a}, J^{c d}\right]=\eta^{a c} P^{d}-\eta^{a d} P^{c},} \\
& {\left[P^{a}, P^{b}\right]=0,} \\
& {\left[J^{a b}, J^{c d}\right]=\eta^{a d} J^{b c}+\eta^{b c} J^{a d}-\eta^{b d} J^{a c}-\eta^{a c} J^{b d} .}
\end{aligned}
$$

If gravity is not present, special theory of relativity forces all the field theories to be invariant under the global action of the Poincaré group. It would be a tempting idea to derive gravitational interaction by demanding this symmetry to hold locally. Then, gravity would emerge from special relativity in much the same way as electromagnetism emerges from the invariance of a matter Lagrangian under the change of phase. In order to construct the covariant differential, one needs to introduce the $\operatorname{Lie}(\mathcal{P})$-valued one-form

$$
\mathbb{A}=\frac{1}{2} \omega_{a b} J^{a b}+\Gamma_{a} P^{a}
$$

where $\omega_{a b}=-\omega_{b a}$ and $\Gamma_{a}$ are one-forms ${ }^{5}$ on $\mathcal{M}$. Under gauge transformations II.4, these one-forms transform as

$$
\omega^{\prime}=\Lambda \omega \Lambda^{-1}-d \Lambda \Lambda^{-1}, \quad \Gamma^{\prime}=\Lambda \Gamma-\omega^{\prime} a-d a,
$$

which can be easily verified for infinitesimal transformations (use (II.6)). Here $\omega$ is a matrix with entries $\omega^{a}{ }_{b}$ and $\Gamma$ a column matrix with entries $\Gamma^{a}$. It is now time to recall that we aim to formulate the theory of gravitational interaction, which ought to be bound up with the geometry of space-time, according to Einstein's idea. The first equation of (II.8) is simply the transformation rule for connection one-forms under the change of an orthonormal frame of vector fields. Indeed, orthonormal frames on Lorenzian manifold are connected by local Lorenz transformations. Note also that the antisymmetry $\omega_{a b}=-\omega_{b a}$ means metricity of the resulting space-time connection. Unfortunately, we do not have a metric given apriori on space-time, after we have disposed of the flat Minkowski's one. Hence, we need to provide somehow the space-time with metric structure, preferably via the introduction of a cotetrad field $e$ (it has long been known that tetrads are necessary to include fermions in the theory of gravity [2]). The transformation formula for $e$, compatible with the one for $\omega$ (II.8), would be $e^{\prime}=\Lambda e$ (think of $e$ as a column of one-forms $\left.e^{a}=e_{\mu}^{a} d x^{\mu}\right)$. Therefore one cannot just adopt the translational gauge field $\Gamma$ as representing cotetrad. The solution is to introduce a vector-valued zero-form $y$ (a column of functions on $\mathcal{M}$ ), which transformas under the local Poincaré transformation $(\Lambda, a)$ according to

$$
y^{\prime}=\Lambda y+a
$$

\footnotetext{
${ }^{4}$ We will not discuss parity and time reversal transformations in this article, hence one can think of $\Lambda$ as a proper ortochronus Lorenz matrix.

${ }^{5}$ Here $\mathcal{M}$ is the space-time manifold, which will no longer be the Minkowski space $M$ in the presence of gravity.
} 
and then introduce the cotetrad

$$
e:=\Gamma+D y, \quad D y=d y+\omega y .
$$

$D$ will always denote the Lorenz covariant derivative (see Appendix VII). Then from (II.8) and (II.9) it follows that $e^{\prime}=\Gamma^{\prime}+d y^{\prime}+\omega^{\prime} y^{\prime}=\Lambda e$, as desired. Although the new field $y$ can seem to have been introduced ad hoc, it can be given a natural geometric interpretation in the language of fibre bundles [20, as well as the physical meaning 22] 25] 29. What is more, if the Lagrangian four-form depends on $y$ and $\Gamma$ only via the cotetrad $e$, one is free to acknowledge $e$ as a fundamental field and forget about its origin. Indeed, the variation of such a Lagrangian would be

$$
\delta \mathfrak{L}=\delta e^{a} \wedge \frac{\delta \mathfrak{L}}{\delta e^{a}}+\delta \omega^{a b} \wedge \frac{\delta \mathfrak{L}}{\delta \omega^{a b}}+\delta \phi \wedge \frac{\delta \mathfrak{L}}{\delta \phi},
$$

$\phi$ representing matter fields. Since $\delta e^{a}=\delta \Gamma^{a}+D \delta y^{a}+\delta \omega^{a}{ }_{b} y^{b}$, we finally get

$$
\begin{aligned}
\delta \mathfrak{L} & =\delta \Gamma^{a} \wedge \frac{\delta \mathfrak{L}}{\delta e^{a}}-\delta y^{a} D\left(\frac{\delta \mathfrak{L}}{\delta e^{a}}\right) \\
+\delta \omega^{a b} & \wedge\left(y_{b} \frac{\delta \mathfrak{L}}{\delta e^{a}}+\frac{\delta \mathfrak{L}}{\delta \omega^{a b}}\right)+\delta \phi \wedge \frac{\delta \mathfrak{L}}{\delta \phi}+d\left(\delta y^{a} \frac{\delta \mathfrak{L}}{\delta e^{a}}\right) .
\end{aligned}
$$

Comparing III.12 with (II.11) one can see that promoting $e$ to the fundamental field, instead of $\Gamma$ and $y$, do not influence the resulting system of field equations. Having the cotetrad introduced, we can define the torsion two-form $Q^{a}:=D e^{a}=\frac{1}{2} T_{b c}^{a} e^{b} \wedge e^{c}$. Then using (II.7) and relations (II.6) one finds that

$$
\mathbb{F}=\frac{1}{2} \Omega_{a b} J^{a b}+\left(Q_{a}-\Omega_{a}{ }^{b} y_{b}\right) P^{a},
$$

where $\Omega_{b}^{a}{ }_{b}:=d \omega^{a}{ }_{b}+\omega^{a}{ }_{c} \wedge \omega^{c}{ }_{b}=\frac{1}{2} R^{a}{ }_{b c d} e^{c} \wedge e^{d}$ is the curvature two-form. One can check that $\mathbb{F}$ obeys (II.5) (the Bianchi identity appears to be equivalent to $d \Omega+[\omega, \Omega]=0, D Q=\Omega e)$.

By adopting $\mathfrak{L}=\mathfrak{L}_{G}+\mathfrak{L}_{m}$ as a Lagrangian, where $\mathfrak{L}_{G}=-\frac{1}{4 k} \epsilon_{a b c d} e^{a} \wedge e^{b} \wedge \Omega^{c d}$ represents gravitational part and $\mathfrak{L}_{m}$ the matter part (here $k=8 \pi G$, where $G$ is the gravitational constant), one recovers the field equations of the Einstein-Cartan theory of gravity

$$
\begin{aligned}
& \frac{\delta \mathfrak{L}_{G}}{\delta e^{a}}+\frac{\delta \mathfrak{L}_{m}}{\delta e^{a}}=0 \quad \Leftrightarrow \quad G^{a}{ }_{b}:=R^{a}{ }_{b}-\frac{1}{2} R \delta_{b}^{a}=k t_{b}{ }^{a} \\
& \frac{\delta \mathfrak{L}_{G}}{\delta \omega^{a b}}+\frac{\delta \mathfrak{L}_{m}}{\delta \omega^{a b}}=0 \quad \Leftrightarrow \quad T^{c a b}-T^{a} \eta^{b c}+T^{b} \eta^{a c}=k S^{a b c} \\
& \frac{\delta \mathfrak{L}_{m}}{\delta \phi}=0
\end{aligned}
$$

where $R_{b}^{a}:=\eta^{a c} R_{c d b}^{d}, R:=R_{a}^{a}, T^{a}:=T_{b}^{b a}$ and the dynamical definitions of energy-momentum and spin density tensors on Riemann-Cartan space are

$$
t_{a b} e^{b}:=-\star \frac{\delta \mathfrak{L}_{m}}{\delta e^{a}}, \quad S^{a b c} e_{c}:=2 \star \frac{\delta \mathfrak{L}_{m}}{\delta \omega_{a b}} .
$$

Here $\star$ is the Hodge star of the cotetrad-induced metric $g=\eta_{a b} e^{a} \otimes e^{b}$ (see Appendix VII). We have not yet explained how to construct the matter Lagrangian $\mathfrak{L}_{m}$ from its flat space counterpart. This issue appears to be problematic and will be considered in the following section.

\section{COUPLING GRAVITY TO MATTER FIELDS - STANDARD APPROACH}

Let (II.1) denote the action functional of a classical field theory in Minkowski space $M$. It is well known that the transformation

$$
\mathcal{L} \rightarrow \mathcal{L}^{\prime}=\mathcal{L}+\partial_{\mu} V^{\mu}
$$

of the Lagrangian density changes $\mathfrak{L}$ by a differential

$$
\left.\partial_{\mu} V^{\mu} d^{4} x=£_{V} d^{4} x=-\star d x_{\mu} \wedge d V^{\mu}=d(V\lrcorner d^{4} x\right),
$$


where $£$ denotes the Lie derivative, $\lrcorner$ the internal product, $\star$ is the Hodge star of the flat Minkowski metric and $x^{\mu}$ are inertial coordinates on $M$. Not only does this transformation not change the field equations generated by $S$, but it also leaves the integrated energy and momenta obtained via Noether procedure invariant 8 . Despite the more subtle behavior of the spin density tensor [8], the transformation still seems to be a true symmetry of the theory. We wish now to introduce a new interaction. A reasonable consistency condition would be to require the resulting theory not to depend on whether we have added a divergence to the initial Lagrangian density or not. For the sake of simplicity, let us consider an example of the Dirac field. The most frequently used Lagrangian four-form is

$$
\begin{aligned}
& \mathfrak{L}_{F 0}=-i\left(\star d x_{\mu}\right) \wedge \bar{\psi} \gamma^{\mu} d \psi-m \bar{\psi} \psi d^{4} x \\
& =\bar{\psi}\left(i \gamma^{\mu} \partial_{\mu}-m\right) \psi d^{4} x .
\end{aligned}
$$

Here $\gamma^{\mu}$ are the Dirac matrixes obeying $\gamma^{\mu} \gamma^{\nu}+\gamma^{\nu} \gamma^{\mu}=2 \eta^{\mu \nu}$ and $\bar{\psi}:=\psi^{\dagger} \gamma^{0}$, where $\psi^{\dagger}$ is a Hermitian conjugation of a column matrix (think of $\psi$ as a column of four complex-valued functions on space-time). In the spirit of the conventional Yang-Mills theory, we should merely perform the replacement (II.3) to 'turn on' the interaction. Explicitly,

$$
d \psi \rightarrow d \psi+\mathbb{A} \psi, \quad d \bar{\psi} \rightarrow d \bar{\psi}+\bar{\psi} \gamma^{0} \mathbb{A}^{\dagger} \gamma^{0}
$$

Consider addition of a divergence of a vector field of the form

$$
V^{\mu}=\bar{\psi} B^{\mu} \psi, \quad B^{\mu}=a \gamma^{\mu}+b \gamma^{\mu} \gamma^{5}
$$

to the initial Lagrangian density. Here $a$ and $b$ are arbitrary complex numbers. This form was chosen so that the new Lagrangian was equally 'reasonable' as the original one (quadratic in fields, invariant under global proper Poincaré transformations).Under the replacement (III.4), the differentials $d V^{\mu}$ transform as

$$
d \bar{\psi} B^{\mu} \psi+\bar{\psi} B^{\mu} d \psi \rightarrow d V^{\mu}+\bar{\psi}\left(\gamma^{0} \mathbb{A}^{\dagger} \gamma^{0} B^{\mu}+B^{\mu} \mathbb{A}\right) \psi
$$

In the case of non-gravitational interactions, the gauge groups are unitary (hence $\mathbb{A}$ is anty-hermitian) and their representations do not act on the spinor indedices of $\psi$, which means that $\mathbb{A}$ commutes with $\gamma^{0}$ and $B^{\mu}$. Next, using $\gamma^{0} \gamma^{0}=1$, we can conclude that $d V^{\mu}$ remains unchanged and III.2 is still a differential. Hence, the two equivalent non-interacting theories give rise to the equivalent theories with interaction.

Let us now turn to gravity. The Lagrangian four-form III.3 is invariant under the global action of the Poincaré group

$$
\begin{aligned}
& x \rightarrow x^{\prime}=\Lambda x+a, \quad \psi \rightarrow \psi^{\prime}=S(\Lambda) \psi, \\
& S(\Lambda(\varepsilon)):=\exp \left(-\frac{i}{4} \varepsilon_{\mu \nu} \Sigma^{\mu \nu}\right), \Sigma^{\mu \nu}:=\frac{i}{2}\left[\gamma^{\mu}, \gamma^{\nu}\right]
\end{aligned}
$$

(use the identity $\gamma^{0} S^{\dagger}(\Lambda) \gamma^{0}=S^{-1}(\Lambda)$ to check this invariance). In order to make this symmetry local, it is not sufficient to perform the substitution (III.4). Now we pass from Minkowski space $M$ to the Riemann-Cartan manifold $\mathcal{M}(\omega, e)$ - the manifold with the metric structure (described by $e$ ) and the metric-compatible connection. We should therefore replace the basis of one-forms $d x^{\mu}$ of $M$ by the cotetrad basis $e^{a}$ of $\mathcal{M}$ and use the Hodge star operator $\star$ adapted to $\mathcal{M}$. The resulting Lagrangian four-form is

$$
\begin{aligned}
& \tilde{\mathfrak{L}}_{F 0}=-i\left(\star e_{a}\right) \wedge \bar{\psi} \gamma^{a} D \psi-m \bar{\psi} \psi \epsilon, \\
& D \psi=d \psi-\frac{i}{4} \omega_{a b} \Sigma^{a b} \psi
\end{aligned}
$$

(the matrixes $\gamma^{a}, a=0, \ldots, 3$ are just the same as $\left.\gamma^{\mu}, \mu=0, \ldots, 3\right)$. Here $\epsilon=e^{0} \wedge e^{1} \wedge e^{2} \wedge e^{3}$ is the canonical volume element on $\mathcal{M}$.

In more general terms, for a flat space field theory of a field $\phi$, which is to be adapted to the Riemann-Cartan manifold $\mathcal{M}$ with the metric $g$ and the metric-compatible connection $\nabla$, the procedure thus described amounts to the passage

$$
\mathcal{L}\left(\phi, \partial_{\mu} \phi, \ldots\right) \mathrm{d}^{4} x \longrightarrow \mathcal{L}\left(\phi, \nabla_{\mu} \phi, \ldots\right) \epsilon,
$$

where $\epsilon=\sqrt{|\operatorname{det} g|} d^{4} x$ is the canonical volume four-form, $\operatorname{det} g$ being the determinant of the matrix of components $g_{\mu \nu}=g\left(\partial_{\mu}, \partial_{\nu}\right)$ of the metric tensor. The dots correspond to the possibility of $\mathcal{L}$ to depend on higher derivatives of 
fields. Here $\nabla_{\mu} \phi$ denotes the appropriately defined covariant derivative of $\phi$ with respect to the connection $\nabla$ of $\mathcal{M}$ (the details depend on the particular field $\phi$ ). This procedure will be referred to as the minimal coupling procedure (MCP) for the gravitational interaction.

From the form of the covariant derivative $D \psi$ in (III.7) one can read out the generators of the relevant representation $\rho$ of the Poincaré group and find the representation itself

$$
P^{a}=0, \quad J^{a b}=-\frac{i}{2} \Sigma^{a b}, \quad \rho(\Lambda, a)=S(\Lambda) .
$$

One can find out that they satisfy the relations $\left(\mathrm{II} .6\right.$ and that $D^{\prime} \psi^{\prime}=S(\Lambda) D \psi$, on account of the first equation of (II.8). Hence, the Lagrangian four-form (III.7) is invariant under the local action of the gauge group, as desired. In the most conventional approach to the Poincaré gauge theory of gravity with fermions, which we follow in this article, the translational part of the Poincaré group is realized trivially. The possibility and consequences of a nontrivial realization of translations were addressed in [22] 26] 27] 28.

Let us now consider the effect of the transformation III.1, performed on the initial Lagrangian, on the final Lagrangian four-form on Riemann-Cartan manifold. We shall consider the vector field of the form (III.5). It is straightforward to check that the following Leibniz rule applies

$$
(D \bar{\psi}) B^{a} \psi+\bar{\psi} B^{a} D \psi=d\left(\bar{\psi} B^{a} \psi\right)+\omega^{a}{ }_{b}\left(\bar{\psi} B^{b} \psi\right)
$$

(use $\left[\gamma^{a}, \Sigma^{b c}\right]=4 i \eta^{a[b} \gamma^{c]}$ ). One can next decompose the connection $\omega$ into the Levi-Civita part and the part determined by torsion (see [8] for this kind of calculations) and finally conclude, that the change in the resulting Lagrangian four-form on $\mathcal{M}$ will be

$$
d(V\lrcorner \epsilon)-T_{a} V^{a} \epsilon
$$

where $T^{a}$ is the torsion trace vector introduced in $($ II.13). Within the framework of classical general relativity, where the torsion of the connection is assumed to vanish, the result would be again a differential. In Einstein-Cartan theory the torsion is determined by the spin of matter via the second equation of (II.13) and does not vanish in general. Hence, the equivalent theories of the Dirac field in flat space can lead to the non-equivalent theories with gravitation.

Let us ignore this problem for a moment and look at the field equations generated by (III.7) resulting from variation with respect to $\psi$ and $\bar{\psi}$. The variational derivatives are

$$
\begin{aligned}
\frac{\delta \tilde{\mathfrak{L}}_{F 0}}{\delta \bar{\psi}} & =\left(i \gamma^{a} D_{a} \psi-m \psi\right) \epsilon, \\
\frac{\delta \tilde{\mathfrak{L}}_{F 0}}{\delta \psi} & =\left(-i \gamma^{a} \overline{D_{a} \psi}-m \bar{\psi}-i T_{a} \bar{\psi} \gamma^{a}\right) \epsilon,
\end{aligned}
$$

where $D_{a} \psi$ denotes the $a$-th component of a one-form $D \psi$ in the cotetrad basis: $D \psi=\left(D_{a} \psi\right) e^{a}$. To derive this results, it is useful to know the identity $D\left(\star e_{a}\right)=T_{a} \epsilon$. Contrary to what would be expected from the Dirac field, calculating the variational derivative with respect to $\bar{\psi}$ and equating it to zero yields the equation that is not equivalent to the one obtained by varying with respect to $\psi$. For $\mathfrak{L}_{F 0}$, the equivalence of the corresponding equations follows from the fact that $\mathfrak{L}_{F 0}$ differs by divergence from the real Lagrangian four-form

$$
\mathfrak{L}_{F R}=-\frac{i}{2}\left(\star d x_{\mu}\right) \wedge\left(\bar{\psi} \gamma^{\mu} d \psi-\overline{d \psi} \gamma^{\mu} \psi\right)-m \bar{\psi} \psi d^{4} x .
$$

But this is no longer the case for $\tilde{\mathfrak{L}}_{F 0}$. The commonly accepted solution to this problem is to adopt III.11) as an appropriate flat space Lagrangian. Then the application of MCP yields

$$
\tilde{\mathfrak{L}}_{F R}=-\frac{i}{2}\left(\star e_{a}\right) \wedge\left(\bar{\psi} \gamma^{a} D \psi-\overline{D \psi} \gamma^{a} \psi\right)-m \bar{\psi} \psi \epsilon
$$

and the field equations obtained by varying with respect to $\bar{\psi}$ and $\psi$ are

$$
\begin{aligned}
& i \gamma^{a} D_{a} \psi-m \psi+\frac{i}{2} \gamma^{a} T_{a} \psi=0, \\
& -i \gamma^{a} \overline{D_{a} \psi}-m \bar{\psi}-\frac{i}{2} T_{a} \bar{\psi} \gamma^{a}=0
\end{aligned}
$$

Hence, they are equivalent. This choice of Lagrangian served as the basis for physical investigations in numerous papers. But the reality requirement does not fix the theory uniquely. We can next add to $\mathcal{L}_{F R}$ the divergence of a 
vector field of the form (III.5), where now the parameters $a, b$ are required to be real, since we do not want to destroy the reality of the Lagrangian. If we required the Lagrangian to be parity invariant, we would have to set $b=0$. But there are no arguments in favor of choosing a particular value of $a$, except for some speculations concerning the resulting form of the spin density tensor [8]. The nonzero values of the parameters $a$ and $b$ can lead to the meaningful physical effects [8] [19].

Hence, the standard MCP for the Poincaré gauge theory of gravity appears to involve an ambiguity. In the next section we will present possible solutions to the problem.

\section{HOW TO REMOVE THE AMBIGUITY?}

\section{A. Preliminary remarks}

Let $\tilde{\mathcal{L}}$ and $\tilde{\mathcal{L}}^{\prime}$ denote the results of application of MCP III.8 to the flat space Lagrangian densities $\mathcal{L}$ and $\mathcal{L}^{\prime}$, connected by the transformation (III.1). We could expect their difference to be

$$
\tilde{\mathcal{L}}^{\prime}-\tilde{\mathcal{L}}=\nabla_{\mu} V^{\mu}
$$

where $\nabla_{\mu} V^{\nu}$ is the standard abbreviation for the $\nu$-th component of the covariant derivative of $V$ in the direction of a basis vector field $\partial_{\mu}, \nabla_{\mu} V^{\nu}:=\left(\nabla_{\partial_{\mu}} V\right)^{\nu}=\partial_{\mu} V^{\nu}+\Gamma_{\rho \mu}^{\nu} V^{\rho}$, where the connection coefficients in the holonomic basis $\partial_{\mu}$ are defined by $\nabla_{\partial_{\mu}} \partial_{\nu}=\Gamma^{\rho}{ }_{\nu \mu} \partial_{\rho}$. Similarly, the connection coefficients in the tetrad basis are provided by $\nabla_{\tilde{e}_{a}} \tilde{e}_{b}=\Gamma_{b a}^{c} \tilde{e}_{c}$ and their relation to the connection one-forms is $\omega^{a}{ }_{b}=\Gamma_{b c}^{a} e^{c}$. The difference in the corresponding Lagrangian four-forms can be expressed in terms of the cotetrad and the connection one-forms as

$$
\tilde{\mathfrak{L}}^{\prime}-\tilde{\mathfrak{L}}=-\left(\star e_{a}\right) \wedge D V^{a},
$$

where $D V^{a}=d V^{a}+\omega^{a}{ }_{b} V^{b}$ is the usual covariant derivative of a differential zero-form of vectorial type (see Appendix VII).

Note that these equivalent statements IV.1 and $\sqrt{\text { IV.2 }}{ }^{6}$ are not so obvious. One should think of $V$ as composed of the fields of the theory, an example of such reasonable composition for the Dirac theory being provided by (III.5). In order to see what the divergence of $V$ would become after the application of MCP, we have to rewrite it in such a way that the differential operators act directly on fields. Then the derivatives (or differentials) of fields should be replaced by the covariant ones. In the case of the Dirac field, (IV.2) will be true for the vector field of the form (III.5). This is because the Leibniz rule (III.9 holds in this case. In general, this rule would not apply to $V^{a}=\bar{\psi} A^{a} \psi$, with the matrixes $A^{a}$ being different from $B^{a}$ of (III.5). But such $V^{a}$ would not represent a genuine vector field, as its components would transform improperly under Lorenz transformations. In the following, we will only consider genuine vector fields, with correct transformation properties, to which the results (IV.1) and (IV.2) apply.

One can prove that

$$
\nabla_{\mu} V^{\mu}=\stackrel{\circ}{\nabla} V^{\mu}-T_{\mu} V^{\mu}
$$

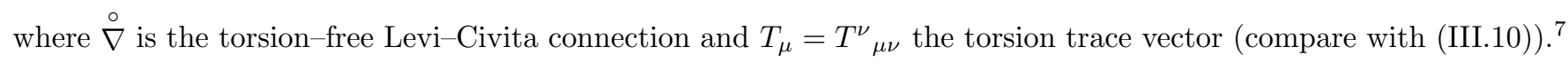

\section{B. Modified volume form approach - Saa's proposal}

After multiplied by the canonical volume element, the first term in (IV.3) becomes a Lie derivative of a four-form (and hence a differential) $\left.\stackrel{\circ}{\nabla}_{\mu} V^{\mu} \epsilon=£_{V} \epsilon=\mathrm{d}(V\lrcorner \epsilon\right)$. This is not the case for the second term. The basic idea of 13 is that we could make the whole expression to be a Lie derivative, if we used another volume element $\tau=f \epsilon$, instead of

\footnotetext{
${ }^{6}$ Use $D V^{a}=\left(\nabla_{b} V^{a}\right) e^{b}$ and $\left(\star e_{a}\right) \wedge e^{b}=-\delta_{a}^{b} \epsilon$ to show this equivalence.

7 The torsion tensor can be defined in terms of the connection $\nabla$ by $T(X, Y)=\nabla_{X} Y-\nabla_{Y} X-[X, Y]$, where $X, Y$ are vector fields and [, ] is the Lie bracket. In the holonomic basis, the components are expressed through the connection coefficients by $T^{\rho}{ }_{\mu \nu}=d x^{\rho}\left(T\left(\partial_{\mu}, \partial_{\nu}\right)\right)=$ $-\Gamma^{\rho}{ }_{\mu \nu}+\Gamma^{\rho}{ }_{\nu \mu}$. The components in the tetrad basis $T_{b c}^{a}=e^{a}\left(T\left(\tilde{e}_{b}, \tilde{e}_{c}\right)\right)$ coincide with those of the torsion two-form $Q^{a}$ introduced in Section IIB
} 
the canonical one. Here, $f$ is a nowhere vanishing function, which should be suitably adapted to the connection (all volume forms on the manifold differ by a nowhere vanishing function). Let us impose the requirement

$$
\nabla_{\mu} V^{\mu} \tau=£_{V} \tau
$$

On account of IV.3), the LHS can be rewritten as $f \stackrel{\circ}{\nabla}_{\mu} V^{\mu} \epsilon-f T_{\mu} V^{\mu} \epsilon$, whereas the RHS can be rewritten as $£_{V}(f \epsilon)=$ $V(f) \epsilon+f £_{V} \epsilon$. Since $\stackrel{\circ}{\nabla}_{\mu} V^{\mu} \epsilon=£_{V} \epsilon$ and $V(f)=V^{\mu} \partial_{\mu} f$, we can see that IV.4 will be satisfied if and only if $T_{\mu}=-\partial_{\mu} \ln f$. The solution to the problem of nonuniqueness of MCP procedure could therefore be to postulate that the torsion trace should be derivable from the potential $-\ln f$ and to promote this potential to the fundamental field of the theory. At the same time, the coupling procedure (III.8) should be slightly modified - instead of the canonical volume form, the connection compatible volume form $\tau=f \epsilon$ ought to be applied. This removes the ambiguity. However, the price is considerably high, as the resulting field equations differ significantly from the usual equations of general relativity, or Einstein-Cartan gravity. Although a lot of interesting effects can be observed on the ground of this theory, such as propagating torsion or coupling gauge fields to torsion without breaking the gauge symmetry [9] 10, some implications of the model seem not to be compatible with observational data [11, 12]. The solution could be to postulate the torsion trace a priori to vanish [13, but such an artificial assumption significantly decreases the elegance of the theory.

\section{Modified connection approach}

Let us recall that the main purpose of the introduction of a covariant derivative (II.3) with $\mathbb{A}$ transforming according to (II.4) was to localize the symmetry. If we used the modified covariant derivative

$$
\stackrel{\mathbb{B}}{D} \phi=D \phi+\mathbb{B} \phi
$$

with $\mathbb{B}$ transforming according to

$$
\mathbb{B}^{\prime}=\rho(g) \mathbb{B} \rho^{-1}(g),
$$

its transformation properties would remain correct, $(\stackrel{\mathbb{B}}{D} \phi)^{\prime}=\rho(g) \stackrel{\mathbb{B}}{D} \phi$, and the local symmetry would be preserved. In general, $\mathbb{B}$ is a $\operatorname{Lin}(\mathcal{V})$-valued one-form on space-time, where $\operatorname{Lin}(\mathcal{V})$ is a set of linear maps of $\mathcal{V}$ into itself, $\mathcal{V}$ being a linear space in which $\phi$ takes its values. Such an additional one-form $\mathbb{B}$ appears when a new gauge symmetry is introduced, in addition to the one corresponding to the representation $\rho$. Then $\mathbb{B}$ transforms inhomogenously, according to (II.4), under the action of its own gauge group. Under the action of $\rho, \mathbb{B}$ has to transform according to IV.6), since we do not want to destroy the already existing gauge symmetry.

Our aim is not to introduce a new gauge symmetry, but to exploit the freedom of addition of $\mathbb{B}$ in the Poincaré gauge theory, in order to modify coupling procedure itself. The idea is to construct $\mathbb{B}$ from $\mathbb{A}$ in such a way that the transformation law (IV.6) follows from the one for $\mathbb{A}$ and the resulting modified coupling procedure, emloying (IV.5), is free of the ambiguity. We shall now consider the Dirac field case and we will see that under some reasonable restrictions the solution to the above formulated problem is unique. The most general $g l(4, \mathbb{C})$-valued one-form can be expanded in the basis of matrixes $\mathbf{1}, \gamma^{5}, \gamma^{a}, \gamma^{5} \gamma^{a}, \Sigma^{a b}$,

$$
\mathbb{B}=\chi \mathbf{1}+\kappa \gamma^{5}+\tau_{a} \gamma^{a}+\rho_{a} \gamma^{5} \gamma^{a}+\frac{1}{2} \alpha_{a b} \Sigma^{a b},
$$

where $\chi, \kappa, \tau_{a}, \rho_{a}, \alpha_{a b}=-\alpha_{b a}$ are complex valued one-forms on space-time. Since we do not want to change the original Poincaré gauge fields $\omega_{a b}$, which are contained in $\mathbb{A}=-\frac{i}{4} \omega_{a b} \Sigma^{a b}$, we will require

$$
\operatorname{Im}\left(\alpha_{a b}\right)=0 .
$$

As noted in Section IVA it is important to demand that the covariant derivative obey the Leibniz rule while acting on vector fields composed of spinor fields. We shall require this rule to hold for vector and axial Dirac currents $J_{(V)}^{a}=\bar{\psi} \gamma^{a} \psi, J_{(A)}^{a}=\bar{\psi} \gamma^{a} \gamma^{5} \psi$. Explicitely, we will require that

$$
\begin{aligned}
& (\stackrel{\mathbb{B}}{D} \bar{\psi}) \gamma^{a} \psi+\bar{\psi} \gamma^{a} \stackrel{\mathbb{B}}{D} \psi=d J_{(V)}^{a}+\stackrel{\mathbb{B}}{\omega}^{a}{ }_{b} J_{(V)}^{b}, \\
& (\stackrel{\mathbb{B}}{D} \bar{\psi}) \gamma^{a} \gamma^{5} \psi+\bar{\psi} \gamma^{a} \gamma^{5} \stackrel{\mathbb{B}}{D} \psi=d J_{(A)}^{a}+\stackrel{\mathbb{B}}{\omega}_{b}{ }_{b} J_{(A)}^{b},
\end{aligned}
$$


where $\stackrel{\mathbb{B}}{D} \bar{\psi}=(\stackrel{\mathbb{B}}{D} \psi)^{\dagger} \gamma^{0}$ and $\stackrel{\mathbb{B}}{\omega}_{b}{ }_{b}$ represents a modified connection on the Riemann-Cartan manifold, which will be determined. These equations can be rewritten as

$$
\begin{aligned}
& \gamma^{0} \mathbb{B}^{\dagger} \gamma^{0} \gamma^{a}+\gamma^{a} \mathbb{B}=\beta^{a}{ }_{b} \gamma^{b}, \\
& \gamma^{0} \mathbb{B}^{\dagger} \gamma^{0} \gamma^{a} \gamma^{5}+\gamma^{a} \gamma^{5} \mathbb{B}=\beta^{a}{ }_{b} \gamma^{b} \gamma^{5},
\end{aligned}
$$

where $\beta=\stackrel{\mathbb{B}}{\omega}-\omega$ is the difference between the two connections on $\mathcal{M}$. Multiplying the first of these equations by $\gamma^{5}$ and comparing with the second we can conclude that $\left[\mathbb{B}, \gamma^{5}\right]=0$ and hence

$$
\tau_{a}=\rho_{a}=0 .
$$

After this requirement is imposed, the second equation of (IV.8) follows from the first one, so we will further deal with the first one only. For $a=0$ it yields

$$
\left(\chi+\chi^{*}\right) \gamma^{0}-\left(\kappa+\kappa^{*}\right) \gamma^{5} \gamma^{0}+i\left(\alpha_{0 j}-\alpha_{0 j}^{*}\right) \gamma^{j}+\frac{1}{2}\left(\alpha_{i j}+\alpha_{i j}^{*}\right) \Sigma^{i j} \gamma^{0}=\beta^{0}{ }_{b} \gamma^{b} .
$$

Knowing that $\Sigma^{i j} \gamma^{0}=\varepsilon^{i j k} \gamma^{5} \gamma^{k}$, where $\varepsilon^{123}=1$, we can conclude that

$$
\beta_{0}^{0}=2 \operatorname{Re}(\chi), \quad \operatorname{Re}(\kappa)=0, \quad \beta^{0}{ }_{j}=-2 \operatorname{Im}\left(\alpha_{0 j}\right), \quad \operatorname{Re}\left(\alpha_{i j}\right)=0 .
$$

For $a=k$, where $k=1,2,3$ we get

$$
2 \operatorname{Re}(\chi) \gamma^{k}+2 i\left(\alpha_{0}^{k} \gamma^{0}+\alpha^{k}{ }_{j} \gamma^{j}\right)=\beta_{0}^{k} \gamma^{0}+\beta^{k}{ }_{j} \gamma^{j},
$$

and hence

$$
\beta_{0}^{j}=-2 \operatorname{Im}\left(\alpha_{0}^{j}\right), \quad \beta^{i}{ }_{j}=2 \operatorname{Re}(\chi) \delta_{j}^{i}-2 \operatorname{Im}\left(\alpha^{i}{ }_{j}\right) .
$$

Recalling (IV.7), we can finally conclude that

$$
\beta_{b}^{a}=\lambda \delta_{b}^{a}, \quad \mathbb{B}=\frac{1}{2} \lambda \mathbf{1}+i \mu_{1} \mathbf{1}+i \mu_{2} \gamma^{5},
$$

where $\lambda:=2 \operatorname{Re}(\chi), \mu_{1}:=\operatorname{Im}(\chi), \mu_{2}:=\operatorname{Im}(\kappa)$ are real-valued one-forms. Hence, the resulting new connection on $\mathcal{M}$ appears to be equal

$$
\stackrel{\lambda}{\omega}_{b}^{a}=\omega_{b}^{a}+\lambda \delta_{b}^{a} .
$$

Note the change of labeling - instead of using $\mathbb{B}$, we are now labeling this connection by the real-valued one-form $\lambda$, on which it depends. Note also that the one-forms $\mu_{1}$ and $\mu_{2}$ remain undetermined, but they do not influence the connection on the Riemann-Cartan space. If non-gravitational interactions were considered, the components of these one-forms could be hidden in the gauge fields corresponding to the localization of the global symmetry of the change of phase $\psi \rightarrow e^{i \alpha} \psi$ and the approximate symmetry under the chiral transformation $\psi \rightarrow e^{i \alpha \gamma^{5}} \psi$. Since we do not want to deal here with non-gravitational interactions, we will assume that $\mu_{1}$ and $\mu_{2}$ are equal to zero.

Let us investigate the properties of the new space-time connection (IV.9). It is no longer metric. The coefficients are expressed through the coefficients of the original metric connection by

$$
\stackrel{\lambda}{\Gamma}_{\mu \nu}^{\rho}=\Gamma_{\mu \nu}^{\rho}+\delta_{\mu}^{\rho} \lambda_{\nu}
$$

In a more mathematically oriented language, it could be defined by

$$
\stackrel{\lambda}{\nabla}_{X} f=X(f), \quad \stackrel{\lambda}{\nabla}_{X} Y=\nabla_{X} Y+\lambda(X) Y,
$$

where $f$ is a function and $X, Y$ are vector fields. Then the action on arbitrary tensor field is uniquely determined by the requirements of commutativity with contraction and Leibniz rule. The action on a $(r, s)$-tensorial type differential form is given by

$$
\stackrel{\lambda}{D} T^{a_{1} \ldots a_{r}}{ }_{b_{1} \ldots b_{s}}=D T_{b_{1} \ldots b_{s}}^{a_{1} \ldots a_{r}}+(r-s) \lambda \wedge T_{b_{1} \ldots b_{s}}^{a_{1} \ldots a_{r}}
$$


and the action on a spinor field is

$$
\stackrel{\lambda}{D} \psi=D \psi+\frac{1}{2} \lambda \psi .
$$

The torsion of this connection is

$$
\stackrel{\lambda}{T}^{c a b}=T^{c a b}+\lambda^{a} \eta^{c b}-\lambda^{b} \eta^{c a} .
$$

According to the leading idea, we do not want to introduce a new field, but rather to express the one-form $\lambda$ through the original connection $\omega$ in such a way that the transformation rule for $\omega$ implies the appropriate transformation rule for $\lambda$. According to $\operatorname{IV.6}$, we need to require that $\mathbb{B}^{\prime}=S(\Lambda) \mathbb{B} S^{-1}(\Lambda)$. But for $\mathbb{B}=\frac{1}{2} \lambda \mathbf{1}$ this simply means that $\lambda$ is a scalar. The simplest possible construction which fulfils the above mentioned requirements employs the torsion trace one-form, $\mathbb{T}=T_{\mu \nu}^{\nu} d x^{\mu}$. Let us denote by $\stackrel{+}{\nabla}$ and $\bar{\nabla}$ the connections $\stackrel{\lambda}{\nabla}$ corresponding to the choice $\lambda=\mathbb{T}$ and $\lambda=-\mathbb{T}$. The second of them proved already useful in Einstein-Cartan theory. To see this, note that (IV.11) enables one to rewrite the equation connecting torsion to the spin distribution of matter (II.13) in a particularly simple form

$$
\bar{T}^{c a b}=k \tilde{S}^{a b c}
$$

What is more, one can prove the geometric identity

$$
G^{a b}-G^{b a}=-\bar{\nabla}_{c} \bar{T}^{c a b}
$$

Together with (II.13), these facts allow us to conclude, that

$$
\tilde{t}^{a b}-\tilde{t}^{b a}=\bar{\nabla}_{c} \tilde{S}^{a b c}
$$

if the field equations are satisfied. This formula corresponds to the well-known relation between the canonical energymomentum tensor and spin density tensor in flat space, $t^{\mu \nu}-t^{\nu \mu}=\partial_{\rho} S^{\mu \nu \rho}$.

The connection $\stackrel{+}{\nabla}$ has not yet been employed in Einstein-Cartan theory. We wish to assign an even more significant role to it. We postulate that this is the connection that ought to be used in the procedure of minimal coupling. Then the flat space divergence of a vector field will pass into

$$
\stackrel{+}{\nabla}_{\mu} V^{\mu}=\nabla_{\mu} V^{\mu}+T_{\mu} V^{\mu}=\stackrel{\circ}{\nabla}_{\mu} V^{\mu}
$$

which becomes a differential when multiplied by the canonical volume form $\epsilon$. Here we used (IV.3) and (IV.10). Having the procedure corrected, we do not have to care anymore about the choice of the flat space Lagrangian from the class of equivalence defined by (III.1). All of them will result in the same theory after gravity is included. Unlike Saa's approach, our proposal is very conservative. As we will see below, it leaves the predictions of the Einstein-Cartan theory almost untouched.

\section{THE CONSEQUENCES OF THE APPLICATION OF THE NEW PROCEDURE.}

Having the procedure established, we can apply it to the Dirac Lagrangian (III.11). It is clear that the result will be the same as the one that would be obtained by means of MCP, since

$$
\stackrel{+}{D} \bar{\psi} \gamma^{a} \psi-\bar{\psi} \gamma^{a} \stackrel{+}{D} \psi=D \bar{\psi} \gamma^{a} \psi-\bar{\psi} \gamma^{a} D \psi
$$

Hence, all the predictions of the Einstein-Cartan gravity with fermions, including the gravity-induced axial-axial point fermion interaction with a very small coupling constant, remain valid. The advantage of the new procedure is that we can use any other equivalent flat Lagrangian as a starting point (in particular, we are now allowed to use the simplest one, namely (III.3) ). Hence, after the new procedure is adopted, the vector-vector and axial-vector fermion interactions discussed in [8] do not appear anymore. The predictions of the theory become unique and perhaps will enable experimental verification after technological barriers are smoothed away in the future.

For a scalar field nothing will change, as a covariant derivative of a scalar does not depend on whether we use the connection $\nabla$ or $\stackrel{+}{\nabla}$. 
Let us consider the vector field, described by the flat space Lagrangian density

$$
\mathcal{L}_{\text {vector }}=-\frac{1}{4} F_{\mu \nu} F^{\mu \nu}+m^{2} A_{\mu} A^{\mu}, \quad F_{\mu \nu}=\partial_{\mu} A_{\nu}-\partial_{\nu} A_{\mu},
$$

where $m$ is a real parameter. For $m=0$, the Lagrangian is reduced to the one of the electromagnetic field, whereas $m>0$ corresponds to Proca field. One can define a one-form field $A=A_{\mu} d x^{\mu}$ and rewrite the derivative-dependent term as

$$
-\frac{1}{4} F_{\mu \nu} F^{\mu \nu} \epsilon=-\frac{1}{2} F \wedge \star F, \quad F=d A=d A_{\mu} \wedge d x^{\mu} .
$$

From the last equation it is clear that gravity can be included via MCP in two different ways. One can either acknowledge $A$ as a scalar-valued one-form and assume that the exterior covariant differential is just the usual differential, $D A=d A$, or assume that $A_{\mu}$ is a one-form valued function and do the replacement

$$
d A_{\mu} \wedge d x^{\mu} \rightarrow D A_{a} \wedge e^{a}, \quad D A_{a}=d A_{a}-\omega^{b}{ }_{a} A_{b} .
$$

In the case of Maxwell field, the second procedure would lead to the breaking of gauge invariance. Therefore, for Maxwell field, as well as the non-Abelian gauge fields, the first possibility is usually adopted. In the $m>0$ case both approaches are in principle possible, however the latter one seems more appropriate, as it results in a reasonable form of the spin density tensor ${ }^{8}$. According to the ideas presented in this article, nothing will change in the $m=0$ case (as well as in the case of non-Abelian gauge fields). This is because the exterior covariant differential of a scalar-valued one-form is just the usual differential, independently of the choice of a connection. Hence, as opposed to Saa's theory, the gauge fields do not couple to torsion in our approach. In the $m>0$ case, the usage of $\stackrel{+}{\nabla}$ instead of $\nabla$ makes difference, as we end with the Lagrangian four-form

$$
\begin{aligned}
& \mathfrak{L}_{\text {proca }}=-\frac{1}{2} \stackrel{+}{F} \wedge \star \stackrel{+}{F}+m^{2} A_{a} A^{a} \epsilon, \\
& \stackrel{+}{F}=\tilde{F}-\mathbb{T} \wedge A, \quad \tilde{F}=D A_{a} \wedge e^{a}=d A-A_{a} Q^{a} .
\end{aligned}
$$

Te dynamical spin density tensor is then

$$
\stackrel{+}{S}^{a b c}=A^{a} \stackrel{+}{F}^{c b}-A^{b} \stackrel{+}{F}^{c a}+A_{d}\left(\stackrel{+}{F}^{a d} \eta^{b c}-\stackrel{+}{F}^{b d} \eta^{a c}\right)
$$

where $\stackrel{+}{F}_{a b}=\tilde{F}_{a b}+A_{a} T_{b}-A_{b} T_{a}, \tilde{F}_{a b}=\tilde{e}_{a}\left(A_{b}\right)-\tilde{e}_{b}\left(A_{a}\right)-A_{c} T^{c}{ }_{a b}$. Had we used the usual MCP with the connection $\nabla$, we would have obtained the spin density tensor

$$
S^{a b c}=A^{a} \tilde{F}^{c b}-A^{b} \tilde{F}^{c a} .
$$

Contrary to the Dirac field case, both the spin tensors depend on torsion, which results in the second equation of (II.13) having a more complicated structure. It can still be rewritten as a linear equation for the 24 components of the torsion tensor. However, the corresponding linear operator $L(A)$ may not be invertible for some values of the field $A$, which leads to the occurrence of the well-known torsion singularities [24] in the theory. The determinant of this operator is $\operatorname{det}(L(A))=2\left(2+k A_{a} A^{a}\right)^{3}$ in the case of standard MCP and $\operatorname{det}(L(A))=16\left(1-2 k A_{a} A^{a}\right)^{3}$ if the connection $\stackrel{+}{\nabla}$ is used. These singularities are therefore not removed by our procedure, but they are shifted towards other values of $A$. It is surprising that the values of $A$ for which a singularity may appear are of opposite causal character in these cases.

\section{CONCLUSIONS}

The modified coupling procedure introduced in Section IV C provides a unique method for coupling gravity to other field theories, which is compatible with the Poincaré gauge description of the gravitational interaction. This method

\footnotetext{
8 The first approach leads to vanishing of the dynamical spin density tensor $S^{a b c}$ (see II.13).
} 
is a slight modification of the procedure which could be considered as the most natural choice for a gauge theory, namely MCP. However, as opposed to MCP, the results obtained by this method do not depend on the choice of a flat space Lagrangian from the class of equivalence connected with the possibility of the addition of a divergence. In particular, this makes the predictions of EC gravity with fermions unique. They appear to agree with those derived in earlier accounts for a particular choice of fermionic Lagrangian. The classical theories of scalar field and gauge fields in the presence of gravity do not change, if our approach is adopted. For the massive vector field, the torsion singularities appear to occur for different values of field than in the standard EC treatment. This result is discussed rather as a curiosity, as the set of fundamental matter fields which are present in nature seems not to contain the Proca field.

There is finally an aesthetic argument which makes our procedure yet more appealing. The fundamental motivations underlying the standard minimal coupling procedure leave certain freedom, when applied to gravity. This freedom is precisely employed by us to remove the ambiguity. In the standard treatment of Poincaré gauge theory in which minimal coupling procedure is used, the above mentioned freedom is ignored.

\section{Acknowledgements}

I wish to thank Wojciech Kamiński, Jerzy Lewandowski and Andrzej Trautman for helpful comments and Urszula Pawlik for linguistic corrections. This work was partially supported by the Foundation for Polish Science, Master grant.

\section{APPENDIX 1: NOTATION AND CONVENTIONS}

Throughout the paper $a, b, \ldots$ are orthonormal tetrad indices and $\mu, \nu, \ldots$ correspond to a holonomic frame. For inertial frame of flat Minkowski space, which is both holonomic and orthonormal, we use $\mu, \nu, \ldots$ The metric components in an orthonormal tetrad basis $\tilde{e}_{a}$ are $g\left(\tilde{e}_{a}, \tilde{e}_{b}\right)=\left(\eta_{a b}\right)=\operatorname{diag}(1,-1,-1,-1)$. Lorenz indices are shifted by $\eta_{a b} . \epsilon=e^{0} \wedge e^{1} \wedge e^{2} \wedge e^{3}$ denotes the canonical volume four-form whose components in orthonormal tetrad basis obey $\epsilon_{0123}=-\epsilon^{0123}=1$. The action of a covariant exterior differential $D$ on any $(r, s)$-tensorial type differential $m$-form

$$
T^{a_{1} \ldots a_{r}} b_{1} \ldots b_{s}=\frac{1}{m !} T^{a_{1} \ldots a_{r}} b_{1} \ldots b_{s} \mu_{1} \ldots \mu_{m} d x^{\mu_{1}} \wedge \ldots \wedge d x^{\mu_{m}}
$$

is given by

$$
\begin{aligned}
& D T_{{ }_{1} \ldots b_{s}}^{a_{1} \ldots a_{r}}:=d T^{a_{1} \ldots a_{r}}{ }_{b_{1} \ldots b_{s}} \\
& +\sum_{i=1}^{r} \omega^{a_{i}}{ }_{c} \wedge T_{b_{1} \ldots b_{s}}^{a_{1} \ldots c \ldots a_{r}} \sum_{i=1}^{s} \omega^{c}{ }_{b_{i}} \wedge T^{a_{1} \ldots a_{r}}{ }_{b_{1} \ldots c \ldots b_{s}} .
\end{aligned}
$$

The Hodge star action on external products of orthonormal cotetrad one-forms is given by

$$
\begin{aligned}
& \star e_{a}=\frac{1}{3 !} \epsilon_{a b c d} e^{b} \wedge e^{c} \wedge e^{d}, \quad \star\left(e_{a} \wedge e_{b}\right)=\frac{1}{2 !} \epsilon_{a b c d} e^{c} \wedge e^{d}, \\
& \star\left(e_{a} \wedge e_{b} \wedge e_{c}\right)=\epsilon_{a b c d} e^{d},
\end{aligned}
$$

which by linearity determines the action of $\star$ on any differential form.

\section{APPENDIX 2: THE PRINCIPLES OF EQUIVALENCE AND GENERAL COVARIANCE IN GR}

Note that the principle of general covariance, as formulated by Weinberg on p.91 of [6], is not really of the same physical content as the principle of equivalence, formulated on p.68, although Weinberg claims so. The latter allows for non-minimal couplings. Indeed, if we postulated that the motion of free falling material particle is determined by the equation

$$
\frac{d^{2} x^{\mu}}{d \tau^{2}}+\alpha R \Gamma_{\rho \sigma}^{\mu} \frac{d x^{\rho}}{d \tau} \frac{d x^{\sigma}}{d \tau}+\beta R \frac{d x^{\mu}}{d \tau}=0,
$$


where $\tau(t)=\int_{t_{0}}^{t} \sqrt{\left|g_{\mu \nu}(x(t)) \frac{d x^{\mu}}{d t} \frac{d x^{\nu}}{d t}\right|} d t$ is the proper time, $R$ the curvature scalar, $\Gamma$ represents the Levi-Civita connection and $\alpha, \beta$ are some real constants, which we shall call non-minimal parameters, then the principle of general covariance would not be violated. What about the principle of equivalence? According to Weinberg, it is formulated by the statement that at every space-time point in an arbitrary gravitational field it is possible to chose a 'locally inertial coordinate system' such that, within a sufficiently small region of the point in question, the laws of nature take the same form as in unaccelerated Cartesian coordinate systems in the absence of gravitation. We will call this a weak formulation of the principle of equivalence. Note that in a locally inertial coordinate system the Christoffel symbols will vanish and therefore the freedom of choice of the parameter $\alpha$ is not restricted by such a principle. However, the parameter $\beta$ has to be set to zero.

Now we wish to stress that in fact Weinberg used another, stronger formulation of the equivalence principle to derive the predictions of GR. The stronger principle states that the laws of physics governing the behavior of matter and test particles in the presence of gravity are derivable from those that are valid in the absence of gravity by a well established rule. This rule is that one rewrites the equations of special relativity, originally given in inertial Cartesian coordinates, in arbitrary coordinates. Then the connection coefficients, which assume nonzero values in such coordinates even in flat space, have to be replaced by the connection coefficients of the curved connection on the final Riemannian manifold. In other words, in order to pass from flat to curved space, one should merely replace all the derivatives by the covariant ones. Such a prescription is identical with what we call the minimal coupling procedure. Note that such a principle forces both the parameters $\alpha$ and $\beta$ of (VIII.1) to vanish. It is this strong formulation that enables one to derive the geodesic postulate, as well as other concrete principles of GR, and finally makes the theory experimentally verifiable.

[1] Sitzungsber., Preuss. Akad. der Wissensch. (1925), p. 414.

[2] H. Weil, "A remark on the coupling of gravitation and electron", Phys. Rev. 77, 699 (1950).

[3] C. Yang and R. Mills, "Conservation of isotopic spin and isotopic gauge invariance", Phys. Rev. 96, 191 (1954).

[4] R. Utiyama, "Invariant theoretical interpretation of interaction", Phys. Rev. 101, 1597 (1956).

[5] T. Kibble, "Lorentz invariance and the gravitational field", J. Math. Phys. 2, 212-221 (1960).

[6] S. Weinberg, "Gravitation and cosmology: principles and applications of the general theory of relativity", WILEY (1972)

[7] F. Hehl and B. Data, "Nonlinear spinor equation and asymmetric connection in general relativity", J. Math. Phys. 12, 1334 (1971).

[8] M. Kazmierczak, "Nonuniqueness of gravity induced fermion interaction in the Einstein-Cartan theory", Phys. Rev. D 78, 124025 (2008) arXiv:0811.1932.

[9] A. Saa, "Propagating torsion from first principles", Gen. Rel. Grav. 29, 205 (1997) arXiv:gr-qc/9609011.

[10] A. Saa, "Volume-forms and minimal action principles in affine manifolds", J. Geom. Phys. 15, 102 (1995) arXiv:hepth/9308087.

[11] T. Boyadjiev, P. Fiziev and S. Yazadjiev, "Neutron star in presence of torsion-dilaton field", Class. Quant. Grav. 16, 2359 (1999) arXiv:gr-qc/9803084.

[12] P. Fiziev and S. Yazadjiev, "Solar System Experiments and the Interpretation of Saa's Model of Gravity with Propagating Torsion as a Theory with Variable Plank "Constant" ", Mod. Phys. Lett. A14, 511 (1999) arXiv:gr-qc/9807025].

[13] R. Mosna and A. Saa, "Volume elements and torsion", J. Math. Phys. 46, 112502 (2005) arXiv:gr-qc/0505146].

[14] F. Hehl and P. von der Heyde, "Spin and the structure of space-time", Ann. Inst. Henri Poincaré A19, 179 (1973).

[15] D. Kerlick, "Cosmology and particle pair production via gravitational spin-spin interaction in the Einstein-Cartan-SciamaKibble theory of gravity", Phys. Rev. D 12, 3004 (1975).

[16] H. Rumpf, "Creation of Dirac Particles in General Relativity with Torsion and Electromagnetism I, II, III", Gen. Rel. Grav. 10 509, 525, 647 (1979).

[17] K. Horie, "Geometric Interpretation of Electromagnetism in a Gravitational Theory with Torsion and Spinorial Matter", Ph.D. thesis arXiv:hep-th/9601066.

[18] A. Perez and C. Rovelli, "Physical effects of the Immirzi parameter", Phys. Rev. D 73, 044013 (2006) arXiv:gr-qc/0505081.

[19] M. Kazmierczak, "Einstein-Cartan gravity with Holst term and fermions", (2008) arXiv:0812.1298.

[20] A. Trautman, "On the structure of the Einstein-Cartan equations", Symposia Mathematica 12, 139 (1979).

[21] K. Stelle and P. West, "Spontaneously broken de Sitter symmetry and the gravitational holonomy group", Phys. Rev. D 21, 1466 (1980).

[22] G. Grignani and G. Nardelli, "Gravity and the Poincaré group", Phys. Rev. D 45, 2719 (1992).

[23] F. Hehl, J. McCrea, E. Mielke and Y. Ne'eman, "Metric-affine gauge theory of gravity: field equations, Noether identities, world spinors, and breaking of dilation invariance", Phys. Rep. 258 (1995).

[24] F. Hehl, P. von der Heyde, D. Kerlick and J. Nester, "General relativity with spin and torsion: Foundations and prospects", Rev. Mod. Phys. 48, 393 (1976).

[25] A. Trautman, Czech. J. Phys. B 29, 107 (1979).

[26] M. Leclerc, "Intrinsic momentum in Poincare gauge theory", (2005) [http://arxiv.org/abs/gr-qc/0510098v5arXiv:gr- 
qc/0510098v5].

[27] A. Tiemblo and R. Tresguerres, "Gravitational contribution to fermion masses", Eur. Phys. J. C 42437 (2005).

[28] A. Tiemblo and R. Tresguerres, "Gauge theories of gravity: the nonlinear framework", Recent Res. Devel. Phys. 5 1255 (2004) [http://arxiv.org/abs/gr-qc/0510089arXiv:gr-qc/0510089].

[29] M. Leclerc, "The Higgs sector of gravitational gauge theories", Annals Phys. 321, 708 (2006).

[30] S. Weinberg, "The quantum theory of fields", CUP (1996) 